\title{
Tendinitis calcificante del longísimo del cuello
}

\author{
Acute calcific tendinitis of the longus colli muscles
}

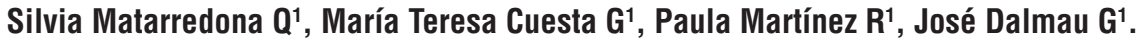

\begin{abstract}
RESUMEN
La tendinitis calcificante prevertebral es una patología benigna y poco frecuente, con una incidencia anual de 0,5 casos por cada 100.000 habitantes. Se presenta un caso de una paciente de 52 años que consultó por cervicalgia, odinofagia y disfonía de 5 días de evolución. Tras una exploración otorrinolaringológica completa se sospechó ocupación del espacio retrofaríngeo, confirmada con pruebas de imagen. Ante estos hallazgos y con la sospecha de absceso retrofaríngeo, se decidió ingreso hospitalario para tratamiento intravenoso. Por discordancia entre la clínica, la TC y los hallazgos analíticos, se solicitó RM cervical, cuya imagen hizo sospechar una tendinitis aguda calcificante del longísimo del cuello. En este trabajo se ha realizado una revisión de la sintomatología, el diagnóstico y el tratamiento de esta entidad. Consideramos importante sospecharla dentro del diagnóstico diferencial de la ocupación del espacio retrofaríngeo para evitar realizar procedimientos innecesarios.
\end{abstract}

Palabras clave: Espacio retrofaríngeo, tendinitis calcificante prevertebral, absceso retrofaríngeo.

\section{ABSTRACT}

Prevertebral calcific tendinitis is a benign and infrequent pathology, with an annual incidence of 0.5 cases per 100,000 habitants. We report the case of a 52-year-old woman that presented with a 5-day history of cervicalgia, odynophagia and dysphonia. Otolaryngological examination and radiological images showed occupation of the retropharyngeal space. The patient was admitted to the hospital for intravenous treatment. A cervical MRI was requested, suggesting an acute calcific tendinitis of the longus coIli muscles. The authors provide a discussion of the clinical findings, diagnosis and treatment of this condition. We consider it to be an important differential diagnosis of a retropharyngeal space occupation, in order to avoid unnecessary procedures.

Key words: Retropharyngeal space, prevertebral calcific tendinitis, retropharyngeal abscess.

\footnotetext{
1 Departamento de Otorrinolaringología, Hospital Universitario Doctor Peset de Valencia, España.
}

Los autores declaran no tener conflictos de interés.

Recibido el 4 de abril, 2019. Aceptado el 10 de julio, 2019. 


\section{INTRODUCCIÓN}

En nuestra especialidad, ante la aparición de ocupación del espacio retrofaríngeo, la primera sospecha diagnóstica suele ser un absceso retrofaríngeo; cuadro potencialmente muy grave, pero no la única opción. Debemos conocer el resto de entidades con clínica similar (dolor cervical, limitación de la movilidad, odinofagia, rigidez y disfagia) $)^{1}$ para poder hacer un correcto diagnóstico diferencial ${ }^{2}$. Algunas de estas entidades incluyen: espondilitis infecciosa, lesiones traumáticas cervicales, tendinitis calcificante del longísimo del cuello, entre otras.

\section{CASO CLÍNICO}

Mujer de 52 años, sin antecedentes de interés, valorada en urgencias por cervicalgia, odinofagia y disfonía de 5 días de evolución. A la exploración se constató febrícula y dolor a la palpación de la musculatura paravertebral bilateral y músculo esternocleidomastoideo derecho. El resto de la exploración otorrinolaringológica fue normal. Los análisis de laboratorio no revelaron alteraciones sugerentes de un proceso infeccioso agudo. En la radiografía cervical se apreció aumento del espacio prevertebral a nivel de C3C6 con rectificación de la lordosis (Figura 1A).

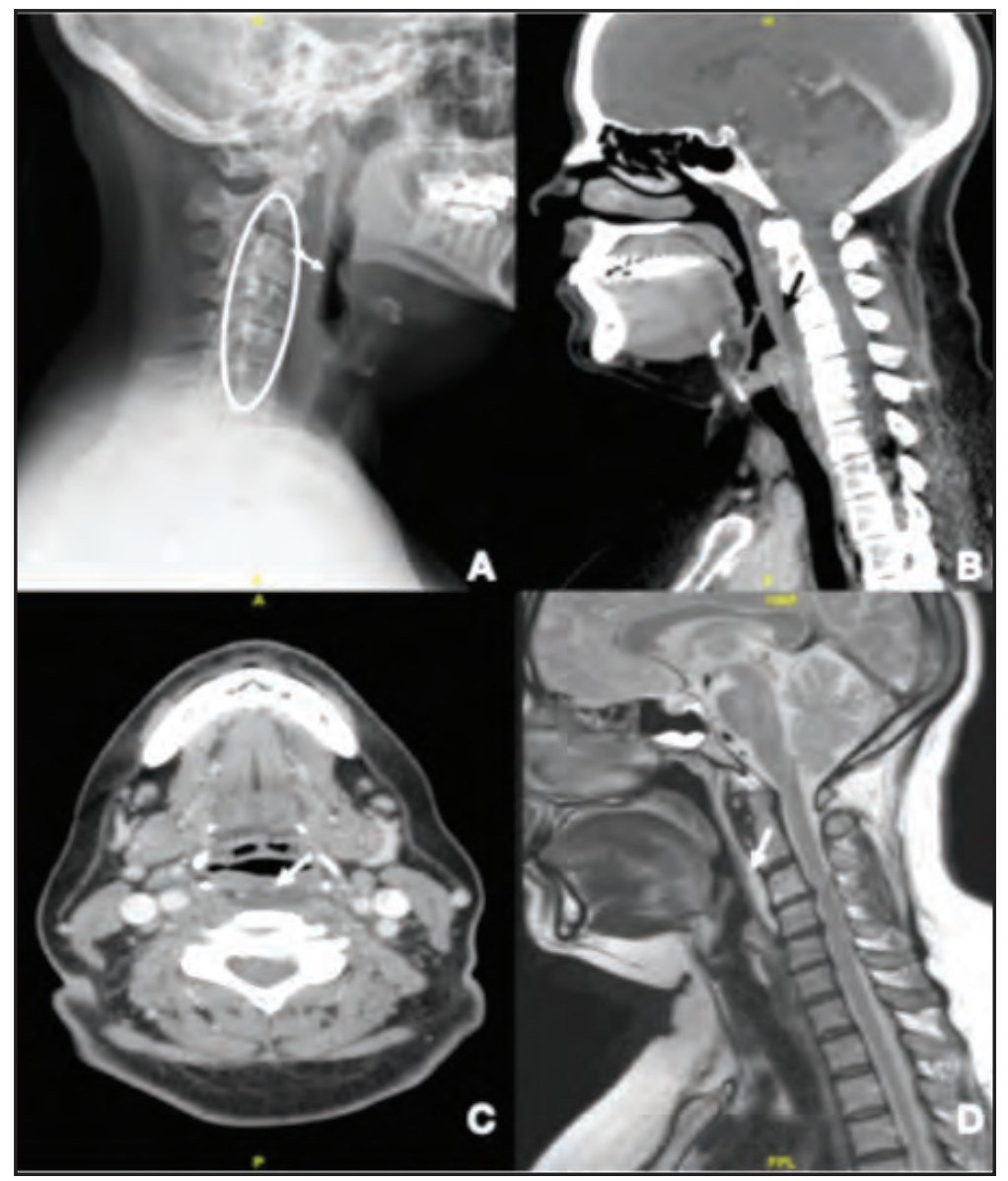

Figura 1. Imágenes de la tendinitis calcificante del longísimo del cuello. (A) Rx cervical: rectificación de la lordosis cervical (elipse) y aumento del espacio prevertebral a nivel C3-C6 (flecha). (B) TC cervical corte sagital: colección hipodensa de 53×11×28 mm en espacio retrofaríngeo a nivel C2-C4 (flecha). (C) TC cervical corte axial: calcificación anterior a C2 (flecha). (D) RM cervical: lesión hiperintensa prevertebral de $75 \times 7 \mathrm{~mm}$ (flecha). 
Debido a lo anterior, se solicitó una tomografía computarizada (TC) cervicotorácica con contraste intravenoso, donde se identificó una imagen hipodensa en el espacio retrofaríngeo, anterior a C2-C4, sin captación de contraste, de 53×11×28 $\mathrm{mm}$ (Figura 1B) y una calcificación anterior a C2 (Figura 1C).

Ante una alta sospecha de absceso retrofaríngeo, se decidió ingreso hospitalario para tratamiento intravenoso. Debido a la discordancia entre la radiología y los hallazgos clínicos y de laboratorio, se realizó una RM cervical, donde se identificó una alteración de la intensidad de señal, de morfología fusiforme en región prevertebral desde el borde inferior del clivus hasta $\mathrm{C} 4$, de $75 \times 7$ mm, sin restricción de la difusión, con realce sólido tras contraste intravenoso; una captación a nivel de las inserciones proximales del longísimo del cuello y calcificación anterior a C2 (Figura 1D). El componente inflamatorio prevertebral, la alteración de la musculatura y la calcificación sugirieron como primera sospecha una tendinitis aguda calcificante del longísimo del cuello por depósito de hidroxiapatita. Ante esta nueva sospecha diagnóstica se decidió suspender la antibioticoterapia intravenosa y mantener una actitud expectante. La paciente evolucionó favorablemente con el tratamiento corticoideo. Tras dos años de seguimiento, refiere que persisten molestias cervicales que trata con antiinflamatorios. En la radiografía cervical persiste calcificación prevertebral y rectificación de la lordosis cervical, pero con una disminución de 5,2 $\mathrm{mm}$ del espacio prevertebral en la radiografía.

\section{DISCUSIÓN}

La tendinitis calcificante prevertebral es una entidad rara, benigna y autolimitada. La incidencia anual es de 0,5 casos por cada 100.000 habitantes $^{3}$. Es más frecuente en pacientes entre 30 y 60 años, y en el sexo femenino ${ }^{4-6}$. La hipótesis etiológica más aceptada es que debido a traumatismos repetidos, se produce una necrosis tisular e isquemia con depósito de cristales de hidroxiapatita cálcica a nivel del músculo longísimo del cuello. Al romperse estos cristales se produce una activación de la respuesta inflamatoria ${ }^{4}$, comparable con lo que ocurre en tendinopatías calcificantes de otras localizaciones. Por ejemplo, en la tendinitis calcificante del hombro se han descrito 4 fases: transformación fibrocartilaginosa del tendón con depósitos focales de calcio, periodo de reposo, reabsorción del calcio que condiciona una respuesta inflamatoria con el cuadro clínico descrito en este caso, fase reparativa con restauración del patrón de colágeno normal ${ }^{7}$.

Esta patología se manifiesta con cervicalgia aguda y contractura muscular refleja junto con disminución del rango de movilidad cervical, y en ocasiones asocia odinofagia lateralizada 0 disfagia ${ }^{5,8}$. Puede presentar febrícula, así como leves alteraciones en la fórmula leucocitaria y la velocidad de sedimentación globular. Radiológicamente, las características que nos deben hacer sospechar esta entidad son: aumento del espacio retrofaríngeo sin realce en anillo y calcificaciones tendinosas en el longísimo del cuello (dato patognomónico) ${ }^{9}$.

El diagnóstico diferencial principal es el absceso retrofaríngeo, ya que en el $75 \%$ de los casos pueden presentar síntomas similares (Tabla 1) ${ }^{10}$.

Tabla 1. Tabla de diagnóstico diferencial de tendinitis calcificante del longísimo del cuello vs absceso retrofaríngeo

\begin{tabular}{|c|c|c|}
\hline & Tendinitis calcificante del longísimo del cuello & Absceso retrofaríngeo \\
\hline Analítica & $\begin{array}{c}\text { Febrícula } \\
\text { 个Leucocitos o N } \\
\text { } V \text { VSG o N }\end{array}$ & $\begin{array}{c}\uparrow \uparrow \uparrow \text { Temperatura } \\
\uparrow \uparrow \uparrow \text { Leucocitos } \\
\text { } \uparrow \text { VSG }\end{array}$ \\
\hline Radiología & $\begin{array}{c}\text { TEspacio retrofaríngeo sin realce en anillo } \\
\text { Calcificaciones tendinosas } \\
\text { en músculo longísimo del cuello }\end{array}$ & 个Espacio retrofaríngeo con realce en anillo \\
\hline Tratamiento & $\begin{array}{l}\text { AINEs + inmovilización } \pm \\
\text { corticoides intravenosos }\end{array}$ & $\begin{array}{c}\text { Antibioticoterapia iv + antiinflamatorios } \\
\text { + vigilancia de la vía aérea superior } \\
\pm \text { drenaje quirúrgico }\end{array}$ \\
\hline
\end{tabular}


La primera línea de tratamiento consiste en antiinflamatorios no esteroideos e inmovilización $y$, en casos graves, corticoides intravenosos ${ }^{4,8}$. El cuadro agudo se resuelve en el transcurso de 1-2 semanas ${ }^{4}$. No suele ser necesario el seguimiento prolongado por el carácter benigno y autolimitado de esta entidad ${ }^{11}$. Aunque, al igual que en las tendinitis calcificantes de otras áreas, la recurrencia de la inflamación aguda es posible, con intervalos de ataque de entre 9 meses y 20 años ${ }^{7}$.

\section{BIBLIOGRAFÍA}

1. Eastwood JD, Hudgins PA, Malone D. Retropharyngeal effusion in acute calcific prevertebral tendinitis: diagnosis with CT and MR imaging. AJNR Am J Neuroradiol 1998; 19: 1789-92.

2. Pellicer V, Pérez C, Magán A. Tendinitis calcificante prevertebral aguda: a propósito de un caso y revisión de la literatura. Rev Esp Cir Ortop Traumatol 2012; 56: 389-92.

3. Horowitz G, Benari 0, Brenner A, et Al. Incidence of retropharyngeal calcic tendinitis (longus colli tendinitis) in the general population. Otolaryngol Head Neck Surg 2013; 148: 955-8.

4. Ring D, Vaccaro AR, Scuderi G, et al. Acute calcic retropharyngeal tendinitis. clinical presentation and pathological characterization. J Bone Joint Surg Am 1994; 76: 1636-42.

5. Omezzine SJ, Hafsa C, Lahmar I, Driss N, Hamza H. Calcific tendinitis of the longus colli: diagnosis by CT. Joint Bone Spine 2008; 75: 90-1.

\section{CONCLUSIÓN}

De manera general, la ocupación radiológica del espacio retrofaríngeo hará sospechar como primera opción un absceso retrofaríngeo. Pero si el estado clínico del paciente y la analítica no concuerdan con esta patología, deben considerarse diagnósticos alternativos. Sí además se observan calcificaciones tendinosas prevertebrales y ausencia de captación en anillo, se debería sospechar la tendinitis calcificante del longísimo del cuello, y así ahorrar al paciente técnicas invasivas y tratamientos antibióticos innecesarios.

6. Tamm A, Jeffery C, Ansari K, Naik S. Acute Prevertebral Calcific Tendinitis. J Radiol Case Rep 2015; 9: 1-5.

7. PaIK NC, LIM CS, Jang HS. Tendinitis of longus colli: computed tomography, magnetic resonance imaging, and clinical spectra of 9 cases. $J$ Comput Assist Tomogr 2012; 36: 755-61.

8. Raggio BS, Ficenec SC, Pou J, Moore B. Acute Calcific Tendonitis of the Longus Colli. Ochsner J 2018; 18: 98-100.

9. Park R, Halpert DE, Baer A, Kunar D, Holt PA. Retropharyngeal calcific tendinitis: case report and review of the literature. Semin Arthritis Rheum 2010; 39: 504-9.

10. Tannebaum RD. Adult retropharyngeal abscess: a case report and review of the literature. J Emerg Med 1996; 14: 147-58.

11. Harnier S, Kuhn J, Harzheim A, Bewermeyer $\mathrm{H}$, Lіммвотн V. Retropharyngeal tendinitis: a rare differential diagnosis of severe headaches and neck pain. Headache 2008; 48: 158-61.

\footnotetext{
Correspondencia: Silvia Matarredona Quiles

Calle Juan de Garay n²1, 46017 ,

Valencia, España

Email: silvia.matarredona@gamil.com
} 